\title{
Cerebral Amyloid Angiopathy-related Inflammation Presenting with Steroid-responsive Higher Brain Dysfunction: Case Report and Review of the Literature
}

Hideya Sakaguchi", Akihiko Ueda, Takayuki Kosaka, Satoshi Yamashita, En Kimura, Taro Yamashita, Yasushi Maeda, Teruyuki Hirano and Makoto Uchino

\begin{abstract}
A 56-year-old man noticed discomfort in his left lower limb, followed by convulsion and numbness in the same area. Magnetic resonance imaging (MRI) showed white matter lesions in the right parietal lobe accompanied by leptomeningeal or leptomeningeal and cortical post-contrast enhancement along the parietal sulci. The patient also exhibited higher brain dysfunction corresponding with the lesions on MRI. Histological pathology disclosed $\beta$-amyloid in the blood vessels and perivascular inflammation, which highlights the diagnosis of cerebral amyloid angiopathy (CAA)-related inflammation. Pulse steroid therapy was so effective that clinical and radiological findings immediately improved.

CAA-related inflammation is a rare disease, defined by the deposition of amyloid proteins within the leptomeningeal and cortical arteries associated with vasculitis or perivasculitis. Here we report a patient with CAArelated inflammation who showed higher brain dysfunction that improved with steroid therapy. In cases with atypical radiological lesions like our case, cerebral biopsy with histological confirmation remains necessary for an accurate diagnosis.
\end{abstract}

Keywords: cerebral amyloid angiopathy, CAA-related inflammation, higher brain dysfunction

\section{Background}

Cerebral amyloid angiopathy (CAA) is a common pathology in the elderly characterized by the deposition of amyloid proteins within the leptomeningeal and cortical arteries [1]. Recently, coexisting inflammations in CAA patients, such as vasculitis or perivasculitis, which clinically resemble central nervous system vasculitis, have been recognized as CAA-related inflammation [2,3]. The inflammation typically responds well to steroid therapy [4], and recent studies have pointed out its similarities with meningoencephalitis induced by immunization to $A \beta$ in Alzheimer disease patients [4-6]. Herein we report a patient with CAA-related inflammation who showed convulsion in the left lower extremity and higher brain

\footnotetext{
* Correspondence: hideya5783@fc.kuh.kumamoto-u.ac.jp
Department of Neurology, Faculty of Life Sciences, Kumamoto University 1-

* Correspondence: hideya5783@fc.kuh.kumamoto-u.ac.jp
Department of Neurology, Faculty of Life Sciences, Kumamoto University 11-1 Honjo, Kumamoto 860-0811, Japan
}

(c) 2011 Sakaguchi et al; licensee BioMed Central Ltd. This is an Open Access article distributed under the terms of the Creative Commons Attribution License (http://creativecommons.org/licenses/by/2.0), which permits unrestricted use, distribution, and reproduction in any medium, provided the original work is properly cited. therapy.

\section{Case presentation}

A 56-year-old man first noticed discomfort in his left lower limb in January 2010. After 7 days, convulsion in the left lower limb suddenly occurred, and he was transported to the emergency hospital. Magnetic resonance imaging (MRI) showed increased white matter intensities in the right parietal lobe on T2-weighted and fluid attenuated inversion-recovery (FLAIR) images. T1-weighted gadolinium (Gd)-enhanced images revealed enhanced leptomeningeal lesions along the parietal sulci (Figure 1A-B). No microhemorrhages were observed with Gradientrecalled echo (GRE)-T2* imaging (1.5T). He was referred to our institution.

On admission, neurological exam showed mild hyperesthesia in the left lower limb and mild hypalgesia in the 

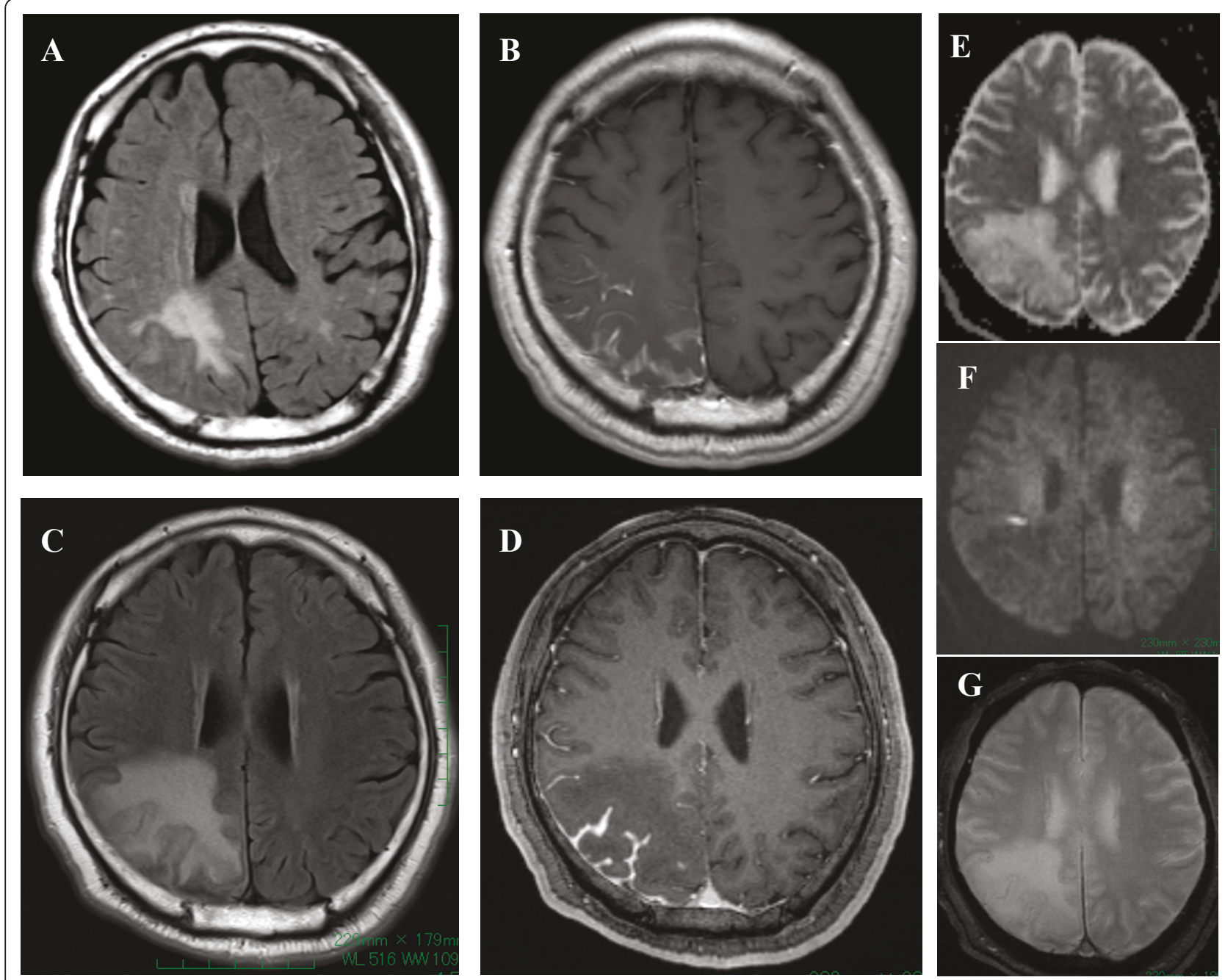

Figure 1 Axial MRI from the referring hospital and on admission to our hospital. MRI findings of FLAIR (A) and T1-weighted image with Gd enhancement (B) from the referring hospital (1.5T). Increased white matter lesions are visible in the right parietal lobe on FLAIR images (A), and a T1-weighted Gd enhanced image revealed abnormal enhanced parenchymal lesions along the parietal sulci (B). On admission, these lesions worsened in both FLAIR (C) and T1-weighted enhanced images (D). High signal intensity in the apparent diffusion coefficient (ADC) map (E) and low signal intensity in the diffusion-weighted image (F) suggested its edematous nature. No microhemorrhages were observed with Gradient recalled echo-T2* imaging (3T) (G).

left crus. No other abnormal findings were present. Biochemical screening tests were generally normal except for serum C-reactive protein $(0.77 \mathrm{mg} / \mathrm{dL})$, soluble interleukin-2 receptor antibody $(462 \mathrm{U} / \mathrm{mL})$, erythrocyte sedimentation rate $(26 / 1 \mathrm{~h}, 72 / 2 \mathrm{~h})$, and carcinoembryonic antigen $(4.5 \mathrm{ng} / \mathrm{mL})$. In the cerebrospinal fluid, protein levels were elevated $(72 \mathrm{mg} / \mathrm{dl})$ and the cell count was mildly elevated $(12 / \mu \mathrm{L})$.

Because a follow-up MRI revealed progression of the white matter lesions and parenchymal enhanced lesions without microhemorrhages (GRE-T2* imaging; 3T) (Figure $1 \mathrm{C}-\mathrm{G}$ ), a brain biopsy was performed in March 2010. Histological pathology showed nonspecific meningoencephalitis involving perivasculitis of the leptomeninges and cortical gray matter (Figure 2A-D).
Starting in April 2010, the patient complained of difficulty with his handwriting. Neuropsychological tests of higher brain functions revealed mild constructional apraxia, line imbalance for words and numbers, difficulty drawing a figure following oral instructions, and problems with visual reproduction. No apathy or dementia was observed.

After the episode, further histological analysis with Congo-red staining disclosed amyloid laden blood vessels. Immunohistochemical staining for $\beta$-amyloid led to the diagnosis of CAA-related inflammation (Figure 2F-G).

Steroid pulse therapy (methylprednisolone $1 \mathrm{~g} /$ day for 3 days) was performed. The abnormal Gd-enhanced findings immediately improved with gradually decreasing FLAIR findings, and the higher brain dysfunctions also gradually resolved (Figure 3). 


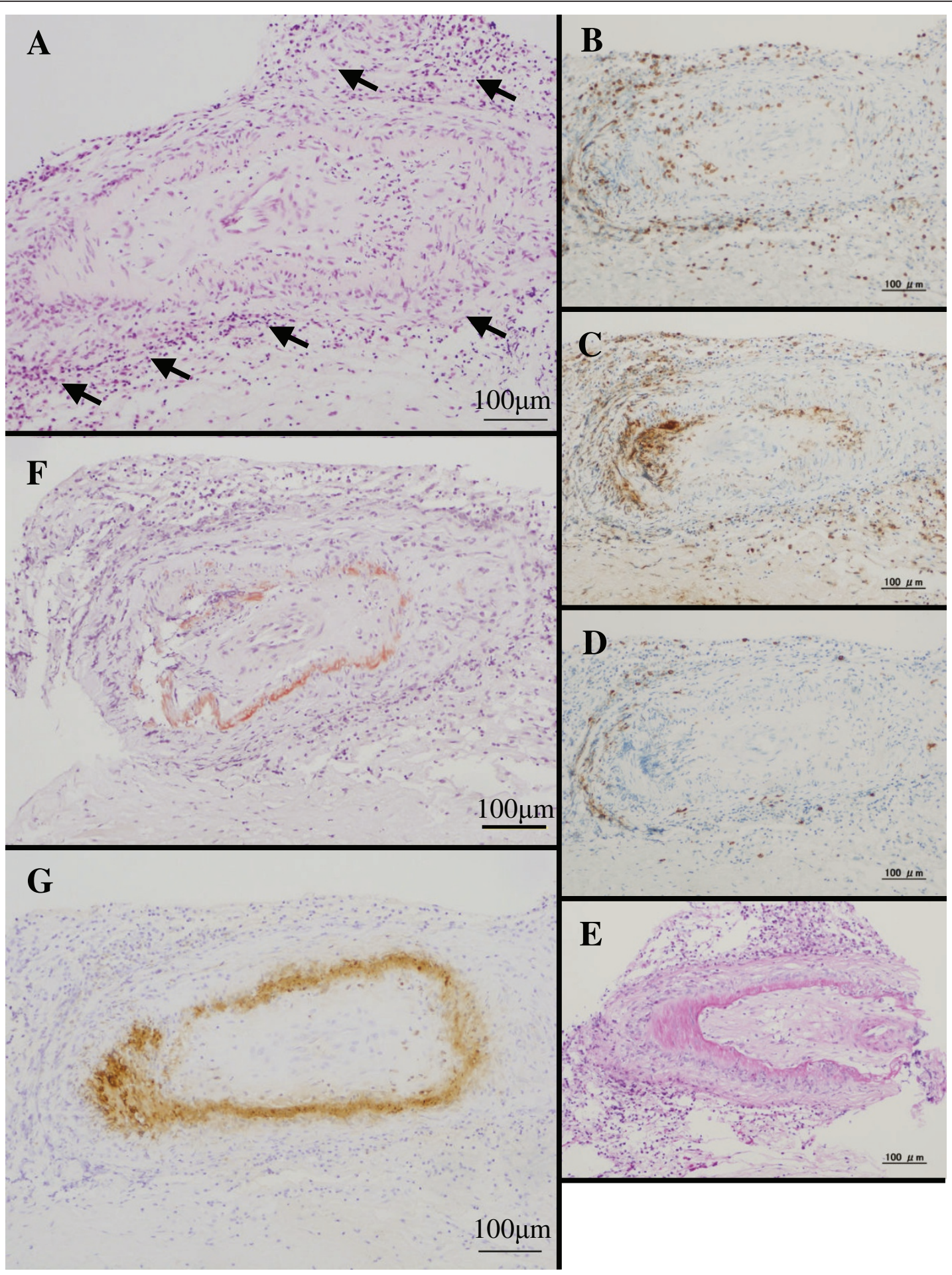

Figure 2 Histological and immune-histological examination of brain biopsy. Microscopic examination showed nonspecific meningoencephalitis involving perivasculitis of leptomeninges (arrows) and cortical gray matter (A). The cellular infiltrate was mainly composed of CD-3-positive T-lymphocytes (B) and CD-68-positive macrophages (C) with minimal CD-20-positive B-lymphocytes (D). PAS staining showed no deposits (E). Congo-red staining revealed amyloid positive blood vessels ( $F$ ); the amyloid was disclosed to be amyloid- $\beta$ by immunohistochemical staining $(G)$. 

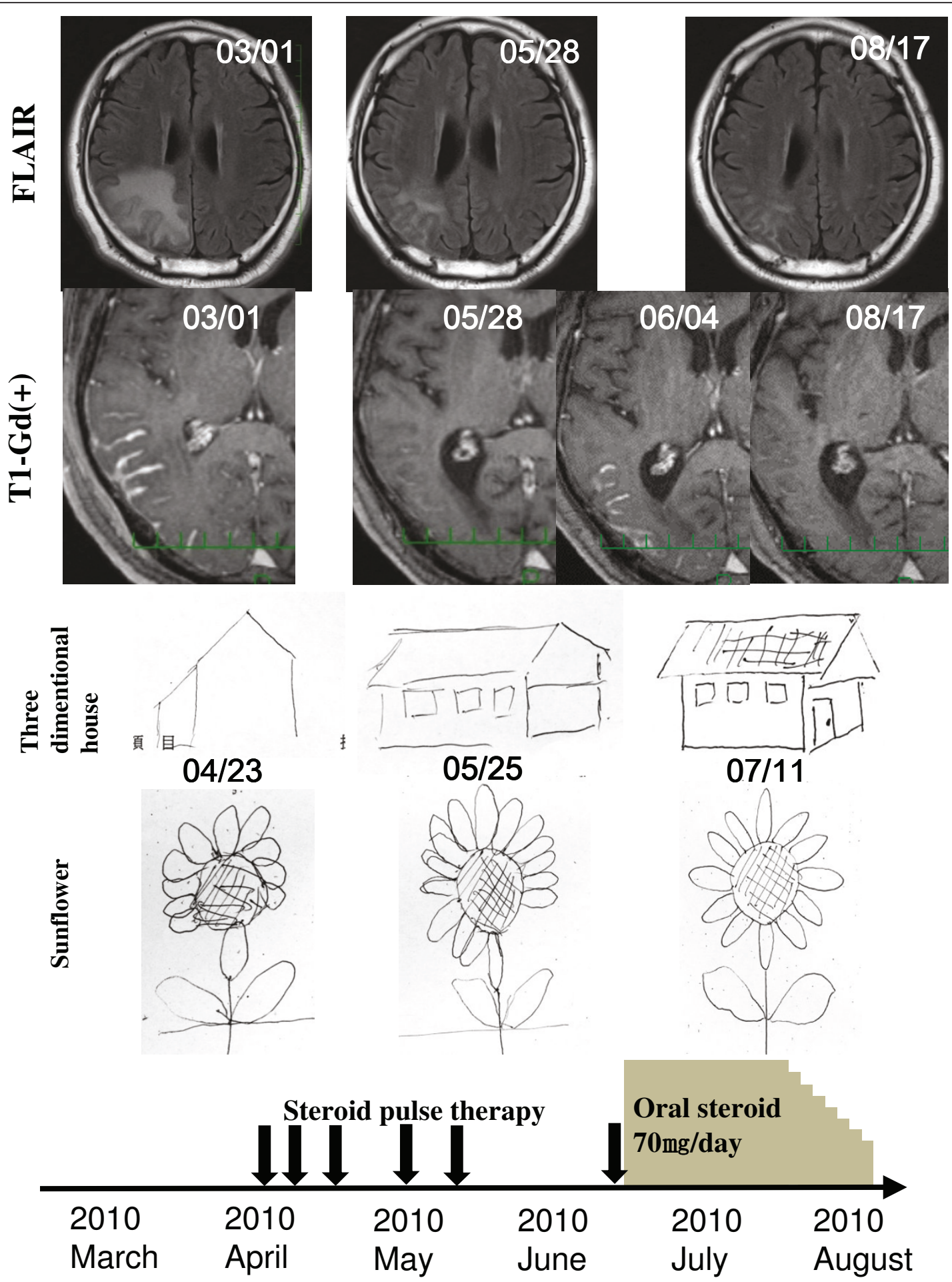

Figure 3 Clinical course of treatment with steroid. Abnormal T1 Gd-enhanced findings immediately improved in the fifth course of steroid pulse therapy, accompanied by a gradual decrease of FLAIR findings and a gradual improvement in higher brain function. As the MRI lesions improved (05/28), the descriptions of the 3D-house and sunflower were made more vivid (05/25). Because T1 Gd-enhanced lesions almost disappeared after the fifth course of the steroid (05/28), we stopped the steroid therapy, and the lesion relapsed (06/04). However, after the initiation of oral steroid therapy, no relapse was observed either clinically or radiologically (08/17). 
After the fifth course of steroid pulse treatment, the T1-enhanced lesions had almost disappeared, and we stopped the treatment. However, 2 weeks later, the lesions had relapsed on a follow-up MRI, although no clinical signs were observed. We performed pulse steroid therapy again, followed by oral methylprednisolone therapy $(70 \mathrm{mg} /$ day). After the oral steroid therapy was initiated, no relapses were observed either clinically or radiologically. Two months later, the oral steroid was tapered at a rate of $5 \mathrm{mg} /$ week, and he was discharged on a regimen of methylprednisolone $30 \mathrm{mg} /$ day.

\section{Discussion}

CAA is defined by the deposition of amyloid proteins within leptomeningeal and cortical arteries, arterioles, and capillaries [1]. Recently, a subset of patients who presented with seizures, subacute cognitive decline, or headaches with hyperintensities on T2-weighted or FLAIR MRI images with microhemorrhages were described as having CAA-related inflammation [2,3]. Neuropathologic examination has generally revealed angiitis of CAA-affected vessels and peripheral inflammation, presenting as vasculitis or perivasculitis [7]. Both pathologic forms can co-exist, and it has been suggested that the prognosis is better for the perivascular type [8]. This inflammation appears to represent an autoimmune response to vascular $\beta$-amyloid deposits. The mechanism by which this immune response occurs is not well understood, although one possible factor is the increased frequency of apolipoprotein $\mathrm{E} \varepsilon 4 / \varepsilon 4$ genotype [9].

The clinical spectrum of CAA-related inflammation is mainly composed of rapidly progressive dementia and seizure. Although the initial presentation of our case was seizure and numbness, the subsequent higher brain dysfunction is uncommon. To clarify how often higher brain dysfunction has been observed, we reviewed previous cases including our case (Table 1) [1,3,4,7-37]. In 64 cases, 10 presented with higher brain dysfunction without encephalopathy or dementia (15.3\%). The most frequent symptom was aphasia (6 cases: $9.3 \%$ ), followed by hemineglect (2 cases: $3.1 \%$ ). One other case was reported of various higher brain dysfunction without mental change or dementia, like our case [23]. In these ten cases with higher brain dysfunction, MRI lesions and the presence of leptomeningeal enhancement were inconsistent, and thus the presentation of higher brain dysfunction was considered to be derived from the observed lesion rather than specific to CAA-related inflammation.

The MRI presentation for CAA-related inflammation was previously described as characterized by large confluent areas of predominantly white matter hyperintense signal on T2-weighted or FLAIR images [34]. These lesions are typically asymmetric and involve one or more cortical lesions without evident preferential laterality. T2- weighted gradient-echo sequence images usually showed multiple scattered cortical or subcortical microhemorrhages [34]. However, these microhemorrhages were not observed in our case, resulting in a delayed diagnosis. In our review, 13 cases were examined by MRI with an echo gradient sequence, and microhemorrhages were not seen in 2 cases including our case (13.3\%). A possible explanation is that the inflammation caused by the immunoreactivity to amyloid might precede the vascular change of cerebellar amyloid angiopathy in some cases, such that microhemorrhages were not observed in radiological exams. This suggests that the gradient-echo sequence image might not be adequate for diagnosis of CAArelated inflammation in all cases. Brain biopsy should be considered if CAA-related inflammation is highly suspected from clinical presentation, even if microhemorrhages were not radiologically observed.

Approximately three quarters of all patients described had a good clinical response to corticosteroid therapy. Additionally, patients presenting with CAA and meningeal enhancement seem to have less progressive disease [29]. In our review, the leptomeningeal enhancement status of 42 patients was mentioned, and the clinical courses of 39 patients were described. Among 19 patients with leptomeningeal enhancement, only one patient died (5.3\%) and the remaining 18 patients survived. However, among the other 20 patients without enhancement, 7 patients died (35\%), suggesting that leptomeningeal enhancement might be a good prognostic factor.

The distinctive pattern of asymmetric MRI lesions in CAA-related inflammation appears to be distinguishable from both non-inflammatory CAA and other causes. This observation raises the possibility that typical MRI findings should prove sufficient to diagnose CAA-related inflammation without necessitating brain biopsy [4]. However, in our case, preoperative imaging did not show the typical microhemorrhages associated with CAA, and the diagnosis could not have been established before biopsy. Therefore, we suggest that cerebral biopsy with histological confirmation remains necessary for an accurate diagnosis.

\section{Conclusion}

We described a patient with CAA-related inflammation whose higher brain functions were dramatically improved by steroid therapy. Because the improvement of cognitive function paralleled resolution of the lesions seen on MRI, this report demonstrates clinically and radiologically progressive improvement of CAA-related inflammation. Our case also suggests the importance of brain biopsy for diagnosis in a case with atypical radiological findings, because correct diagnosis and treatment are crucial for successful recovery and good prognosis. 
Table 1 Review of reported cases of CAA-related inflammation

\begin{tabular}{|c|c|c|c|c|c|c|c|c|c|c|c|}
\hline Reference & $\mathrm{n}$ & Age & Sex & Clinical presentation & & MRI lesion & $\begin{array}{l}\text { Micro bleeds in T2*- } \\
\text { weighted images }\end{array}$ & $\begin{array}{c}\text { MRI } \\
\text { enhanced } \\
\text { lesion }\end{array}$ & Pathology & treatment & Outcome \\
\hline $\begin{array}{l}\text { Greenberg et } \\
\text { al. } 1993 \text { [10] }\end{array}$ & 1 & 72 & $F$ & dementia headache & left & frontal & NA & $(-)$ & vasculitis & NA & NA \\
\hline $\begin{array}{l}\text { Ortiz et al. } \\
1996 \text { [11] }\end{array}$ & 1 & 68 & $\mathrm{~F}$ & headache & right & temporal/parietal & NA & $(-)$ & vasculitis & steroid & NA \\
\hline \multirow[t]{2}{*}{$\begin{array}{l}\text { Fountain et } \\
\text { al. } 1996[12]\end{array}$} & 2 & 66 & M & $\frac{\text { fluent aphasia right }}{\text { hemianopia }}$ & bilateral & temporal/parietal & NA & $(-)$ & $\begin{array}{l}\text { vasculitis } \\
\text { perivasculitis }\end{array}$ & $\begin{array}{c}\text { steroid } \\
\text { cyclophosphamide }\end{array}$ & $\begin{array}{c}\text { alive relapse } \\
(+)\end{array}$ \\
\hline & & 69 & $\mathrm{~F}$ & $\begin{array}{l}\text { headache confusion focal } \\
\text { neurology seizure }\end{array}$ & bilateral & confluent multifocal & NA & NA & vasculitis & $\begin{array}{c}\text { steroid } \\
\text { cyclophosphamide }\end{array}$ & $\begin{array}{c}\text { died relapse } \\
(+)\end{array}$ \\
\hline \multirow{2}{*}{$\begin{array}{l}\text { Anders et al. } \\
1997 \text { [13] }\end{array}$} & 2 & 70 & M & mental status change & right & frontal & NA & NA & vasculitis & NA & NA \\
\hline & & 69 & M & $\begin{array}{c}\text { headache lethargy behavior } \\
\text { change }\end{array}$ & bilateral & white matter & NA & $(+)$ & vasculitis & NA & NA \\
\hline $\begin{array}{l}\text { Fountain et } \\
\text { al. } 1999[14]\end{array}$ & 1 & 71 & M & $\begin{array}{l}\text { headache confusion gait } \\
\text { difficulty left hand apraxia }\end{array}$ & right & temporal/parietal & NA & NA & vasculitis & cyclophosphamide & $\begin{array}{c}\text { alive relapse } \\
(+)\end{array}$ \\
\hline $\begin{array}{l}\text { Scully et al. } \\
2000[15]\end{array}$ & 1 & 63 & $M$ & behavior change ataxia & bilateral & white matter & NA & $(+)$ & perivasculitis & cyclophosphamide & alive \\
\hline $\begin{array}{l}\text { Oide et al. } \\
2002[16]\end{array}$ & 1 & 69 & M & dizziness dementia seizure & bilateral & symmetrical periventricular & NA & NA & vasculitis & $(-)$ & NA \\
\hline \multirow[t]{2}{*}{$\begin{array}{l}\text { Schwab et al. } \\
2003[8]\end{array}$} & 2 & 74 & M & seizure dementia headache & bilateral & multifocal & NA & $(+)$ & perivasculitis & steroid & $\begin{array}{c}\text { alive relapse } \\
(+)\end{array}$ \\
\hline & & 70 & $F$ & dementia headache & right & temporal & NA & $(+)$ & perivasculitis & steroid & $\begin{array}{c}\text { alive relapse } \\
(+)\end{array}$ \\
\hline $\begin{array}{l}\text { Tamargo et } \\
\text { al. } 2003[17]\end{array}$ & 1 & 80 & $F$ & $\begin{array}{c}\text { dementia left-side } \\
\text { hemineglect word finding } \\
\text { difficulty }\end{array}$ & bilateral & left frontal right parietal & NA & $(+)$ & vasculitis & steroid & alive \\
\hline \multirow[t]{2}{*}{$\begin{array}{l}\text { Oh et al. } \\
2004[1]\end{array}$} & 2 & 80 & $\mathrm{~F}$ & Headache aphasia & bilateral & $\begin{array}{l}\text { right parietal/occipital left } \\
\text { frontal }\end{array}$ & NA & $(-)$ & perivasculitis & steroid & alive \\
\hline & & 77 & $M$ & aphasia & left & temporal & NA & $(-)$ & vasculitis & steroid & alive \\
\hline $\begin{array}{l}\text { Safriel et al. } \\
2004 \text { [18] }\end{array}$ & 1 & 49 & $M$ & seizure & right & occipital/temporal & NA & $(-)$ & vasculitis & steroid & alive \\
\hline $\begin{array}{l}\text { Hashizume et } \\
\text { al. } 2004 \text { [19] }\end{array}$ & 1 & 65 & M & $\begin{array}{l}\text { headache left hemianopsia } \\
\text { left-side hemineglect }\end{array}$ & right & temporal/occipital & NA & $(+)$ & vasculitis & $\begin{array}{l}\text { steroid } \\
\text { cyclophosphamide }\end{array}$ & died \\
\hline $\begin{array}{l}\text { Harkness et } \\
\text { al. } 2004 \text { [20] }\end{array}$ & 1 & 72 & $\mathrm{~F}$ & dementia & bilateral & frontal & NA & $(-)$ & vasculitis & no specific therapy & alive \\
\hline $\begin{array}{l}\text { Jacobs et al. } \\
2004 \text { [21] }\end{array}$ & 1 & 81 & F & $\begin{array}{c}\text { confusion Balint's syndrome } \\
\text { agraphia right-left confusion } \\
\text { finger anomia left-side } \\
\text { neglect }\end{array}$ & bilateral & parietal/occipital & NA & $(+)$ & vasculitis & steroid & alive \\
\hline
\end{tabular}


Table 1 Review of reported cases of CAA-related inflammation (Continued)

\begin{tabular}{|c|c|c|c|c|c|c|c|c|c|c|c|}
\hline $\begin{array}{l}\text { Scolding et } \\
\text { al. } 2005[3\end{array}$ & 6 & $69.3^{*}$ & $\begin{array}{l}\text { M } 3 \\
\text { F } 3\end{array}$ & $\begin{array}{c}\text { encephalopathy } 6 \text { focal } \\
\text { neurology } 2 \text { seizure } 1 \\
\text { headache } 2\end{array}$ & NA & $\begin{array}{c}\text { mutifocal } 1 \text { frontal } 1 \text { diffuse } \\
\text { white matter } 1 \text { right occipital } \\
1 \text { left frontal } 1 \text { bilateral } \\
\text { confluent } 1\end{array}$ & NA & $(+) 1(-) 5$ & vasculitis & $\begin{array}{c}\text { steroid } 3 \text { steroid } \\
\text { cyclophosphamide } 2 \\
\text { tumor resection } \\
\text { steroid } 1\end{array}$ & $\begin{array}{l}\text { alive } 4 \\
\text { (relapse NA) } \\
\quad \text { died } 2\end{array}$ \\
\hline $\begin{array}{l}\text { Mikolaenko } \\
\text { et al. 2006 } \\
\text { [22] }\end{array}$ & 1 & 50 & M & seizure & right & frontal & NA & $(+)$ & vasculitis & surgery & alive \\
\hline $\begin{array}{l}\text { Wong et al. } \\
2006 \text { [23] }\end{array}$ & 1 & 79 & $\mathrm{~F}$ & $\frac{\text { higher brain dysfunction }}{\text { fatigue }}$ & right & frontal/temporal/parietal & NA & NA & vasculitis & steroid & $\begin{array}{c}\text { alive relapse } \\
(+)\end{array}$ \\
\hline $\begin{array}{l}\text { Kinnecom et } \\
\text { al. } 2007 \text { [4] }\end{array}$ & 1 & $62.3^{*}$ & $\begin{array}{l}\text { M } 9 \\
\text { F } 3\end{array}$ & $\begin{array}{c}\text { encephalopathy } 9 \text { headache } \\
5 \text { seizure } 7 \text { aphasia } 1 \\
\text { presyncope } 1\end{array}$ & NA & NA & $\begin{array}{l}\text { NA (the presence of } \\
\text { microbleeds are } \\
\text { mentioned but the } \\
\text { proportion is not } \\
\text { mentioned) }\end{array}$ & NA & perivasculitis & $\begin{array}{l}\text { steroid } 9 \text { steroid } \\
\text { cyclophosphamide } 3\end{array}$ & $\begin{array}{l}\text { alive } 11 \\
\text { (relapse }(+) 3 \text { ) } \\
\quad \text { died } 1\end{array}$ \\
\hline $\begin{array}{l}\text { Greenberg et } \\
\text { al. } 2007 \text { [24] }\end{array}$ & 1 & 63 & M & $\begin{array}{l}\text { headache behavioral change } \\
\text { cognitive change }\end{array}$ & bilateral & multiple & NA & $(+)$ & vasculitis & cyclophosphamide & $\begin{array}{l}\text { alive relapse } \\
(+)\end{array}$ \\
\hline $\begin{array}{l}\text { Marotti et al. } \\
2007 \text { [25] }\end{array}$ & 1 & 57 & $\mathrm{~F}$ & headache seizure & bilateral & $\begin{array}{l}\text { frontal/temporal/insular right } \\
\text { thalamus }\end{array}$ & $(+)$ & $(+)$ & vasculitis & seizure control & died \\
\hline $\begin{array}{l}\text { McHugh et } \\
\text { al. } 2007 \text { [26] }\end{array}$ & 1 & 80 & $F$ & $\begin{array}{c}\text { confusion incontinent urine } \\
\text { global aphasia seizure right } \\
\text { hemianopia right } \\
\text { hemiparesis }\end{array}$ & bilateral & frontal & $(+)$ & $(-)$ & $\begin{array}{l}\text { vasculitis } \\
\text { perivasculitis }\end{array}$ & steroid & $\begin{array}{l}\text { alive relapse } \\
\qquad(+)\end{array}$ \\
\hline $\begin{array}{l}\text { Takada et al. } \\
2007 \text { [27] }\end{array}$ & 1 & 69 & $F$ & headache cognitive decline & bilateral & $\begin{array}{l}\text { right frontal/parietal bilateral } \\
\text { parietal/occipital }\end{array}$ & $(+)$ & $(-)$ & vasculitis & steroid & died \\
\hline $\begin{array}{l}\text { Machida et } \\
\text { al. } 2008[28]\end{array}$ & 1 & 69 & $\mathrm{~F}$ & cognitive decline & bilateral & multifocal & $(-)$ & $(+)$ & perivasculitis & steroid & $\begin{array}{l}\text { alive relapse } \\
\qquad(+)\end{array}$ \\
\hline $\begin{array}{l}\text { Salvarani et } \\
\text { al. } 2008 \text { [29] }\end{array}$ & 8 & $63^{*}$ & $\begin{array}{l}\text { M6 } \\
\text { F2 }\end{array}$ & $\begin{array}{l}\text { encephalopathy } 6 \text { focal } \\
\text { neurology } 2 \text { headache } 3 \\
\text { only aphasia with alexia } 1 \\
\end{array}$ & $\begin{array}{l}\text { bilateral } \\
\quad 8\end{array}$ & multifocal & NA & $(+) 5(-) 3$ & vasculitis & $\begin{array}{l}\text { steroid } 3 \text { steroid } \\
\text { cyclophosphamide } 5\end{array}$ & $\begin{array}{l}\text { improved } 6 \\
\text { died } 1 \\
\text { worsened } 1\end{array}$ \\
\hline $\begin{array}{l}\text { Amick et al. } \\
2008 \text { [30] }\end{array}$ & 1 & 79 & $\mathrm{~F}$ & $\begin{array}{l}\text { transient right sided } \\
\text { weakness }\end{array}$ & left & occipital/parietal & NA & $(-)$ & vasculitis & $(-)$ & died \\
\hline $\begin{array}{l}\text { Alcalay et al. } \\
2009 \text { [31] }\end{array}$ & 1 & 92 & $\mathrm{~F}$ & mental status change & bilateral & multifocal & $(+)$ & $(+)$ & $(-)$ & steroid & alive \\
\hline $\begin{array}{l}\text { Daniëls et al. } \\
2009 \text { [32] }\end{array}$ & 1 & 80 & $F$ & $\begin{array}{c}\text { mental status change right } \\
\text { sided hemiparesis dysphasia } \\
\text { seizure }\end{array}$ & bilateral & $\begin{array}{l}\text { left hemisphere right } \\
\text { parietal/occipital }\end{array}$ & $(+)$ & $(-)$ & $(-)$ & steroid & $\begin{array}{l}\text { alive relapse } \\
\qquad(+)\end{array}$ \\
\hline $\begin{array}{l}\text { Greenberg et } \\
\text { al. } 2010 \text { [9] }\end{array}$ & 1 & 87 & $\mathrm{~F}$ & seizure cognitive impairment & bilateral & multifocal & $(+)$ & NA & perivasculitis & steroid & died \\
\hline $\begin{array}{l}\text { Kloppenborg } \\
\text { et al. } 2010 \text { [7] }\end{array}$ & 1 & 74 & M & $\begin{array}{l}\text { increased sleepiness loss of } \\
\text { initiative seizure }\end{array}$ & bilateral & frontal & $(+)$ & $(+)$ & perivasculitis & steroid & alive \\
\hline $\begin{array}{l}\text { Morishige et } \\
\text { al. } 2010 \text { [33] }\end{array}$ & 1 & 78 & $F$ & motor aphasia dementia & left & frontal & NA & $(+)$ & vasculitis & steroid & alive \\
\hline $\begin{array}{l}\text { Savoiardo et } \\
\text { al. } 2010[34]\end{array}$ & 1 & 76 & M & fatigue confusion & bilateral & temporal/occcipital/frontal & $(+)$ & $(-)$ & $(-)$ & steroid & alive \\
\hline
\end{tabular}


Table 1 Review of reported cases of CAA-related inflammation (Continued)

\begin{tabular}{|c|c|c|c|c|c|c|c|c|c|c|c|}
\hline $\begin{array}{l}\text { Cano et al. } \\
2010 \text { [35] }\end{array}$ & 1 & 76 & M & $\frac{\text { transient motor aphasia }}{\text { transient headache }}$ & bilateral & temporal & $(+)$ & NA & $(-)$ & $(-)$ & alive \\
\hline $\begin{array}{c}\text { DiFrancesco } \\
\text { et al. } 2011 \\
{[36]}\end{array}$ & 1 & 68 & M & memory loss mood disorder & bilateral & multifocal & $(+)$ & $(-)$ & NA & steroid & alive \\
\hline \multirow{3}{*}{$\begin{array}{l}\text { Chung et al. } \\
2011 \text { [37] }\end{array}$} & 3 & 83 & $\mathrm{~F}$ & seizure & bilateral & multifocal & NA & NA & vasculitis & steroid & died \\
\hline & & forties & $\mathrm{F}$ & $\begin{array}{c}\text { headache mild hemiparesis } \\
\text { sensory loss }\end{array}$ & right & parietal/occipital & $(+)$ & NA & vasculitis & steroid & $\begin{array}{c}\text { alive } \\
\text { haemorrhage } \\
(+)\end{array}$ \\
\hline & & 72 & M & seizure left-side neglect left & bilateral & multifocal & NA & NA & $\begin{array}{l}\text { vasculitis } \\
\text { perivasculitis }\end{array}$ & $\begin{array}{c}\text { steroid } \\
\text { cyclophosphamide }\end{array}$ & died \\
\hline our case & 1 & 56 & M & $\begin{array}{l}\text { Seizure sensory disturbance } \\
\text { higher brain dysfunction }\end{array}$ & right & parietal & $(-)$ & $(+)$ & perivasculitis & steroid & $\begin{array}{l}\text { alive relapse } \\
\qquad(+)\end{array}$ \\
\hline
\end{tabular}

From the literature, we extracted the cases of CAA-related inflammation in which an MRI was evaluated. If autopsy or biopsy was examined, the cases without inflammation were excluded. All cases satisfy the diagnostic criteria of definite or probable CAA-related inflammation proposed by Chung et al. [37]. In 64 cases, 10 presented with higher brain dysfunction without encephalopathy or dementia (15.3\%). The most frequent symptom was aphasia (6 cases: 9.3\%), followed by hemineglect (2 cases: 3.1\%). One case besides the current presented with various higher brain dysfunction without mental change or dementia [23]. In these 10 cases with higher brain dysfunction, MRI lesions and the presence of leptomeningeal enhancement were inconsistent. Thirteen cases were examined with MRI with an echo gradient sequence, and microhemorrhages were not seen in 2 cases, including our case (13.3\%).

The leptomeningeal enhancement status of 42 patients was mentioned, and the clinical courses of 39 patients were described. Only one patient among 19 patients with leptomeningeal enhancement died (5.3\%): however, 7 of 20 patients without enhancement died (35\%), suggesting that leptomeningeal enhancement might be a factor in good prognosis. *: calculated mean 


\section{Consent}

Written informed consent was obtained from the patient for publication of this case report and any accompanying images. A copy of the written consent is available for review by the Editor-in-Chief of this journal.

\section{List of abbreviations}

A $\beta$ : amyloid $\beta$; ADC: apparent diffusion coefficient; CAA: cerebral amyloid angiopathy; FLAIR: fluid attenuated inversion-recovery; Gd: gadolinium; MRI: magnetic resonance imaging; GRE: gradient-recalled echo.

\section{Acknowledgements}

The authors are very grateful to Professor Hitoshi Takahashi of the Brain Research Institute at the University of Niigata for his expert suggestions regarding pathology.

\section{Authors' contributions}

HS designed this article and direction for investigations and drafted the manuscript. AU, TK, SY, EK, TY, YM, TH, and MU contributed to interpretations of clinical, radiological and pathological details. All authors read and approved the final manuscript.

\section{Authors' information}

All authors are members of the Department of Neurology, Faculty of Life Sciences, Kumamoto University, and TK was also a graduate student of the Brain Research Institute, University of Niigata until March 2011.

\section{Competing interests}

The authors declare that they have no competing interests.

Received: 20 May 2011 Accepted: 14 September 2011 Published: 14 September 2011

\section{References}

1. Oh U, Gupta R, Krakauer JW, Khandji AG, Chin SS, Elkind MS: Reversible leukoencephalopathy associated with cerebral amyloid angiopathy. Neurology 2004, 62(3):494-497.

2. Eng JA, Frosch MP, Choi K, Rebeck GW, Greenberg SM: Clinical manifestations of cerebral amyloid angiopathy-related inflammation. Ann Neurol 2004, 55(2):250-256.

3. Scolding NJ, Joseph F, Kirby PA, Mazanti I, Gray F, Mikol J, Ellison D, Hilton DA, Williams TL, MacKenzie JM, et al: Abeta-related angiitis: primary angiitis of the central nervous system associated with cerebral amyloid angiopathy. Brain 2005, 128(Pt 3):500-515

4. Kinnecom C, Lev MH, Wendell L, Smith EE, Rosand J, Frosch MP, Greenberg SM: Course of cerebral amyloid angiopathy-related inflammation. Neurology 2007, 68(17):1411-1416.

5. Nicoll JA, Wilkinson D, Holmes C, Steart P, Markham H, Weller RO: Neuropathology of human Alzheimer disease after immunization with amyloid-beta peptide: a case report. Nat Med 2003, 9(4):448-452.

6. Orgogozo JM, Gilman S, Dartigues JF, Laurent B, Puel M, Kirby LC, Jouanny P, Dubois B, Eisner L, Flitman S, et al: Subacute meningoencephalitis in a subset of patients with $A D$ after Abeta42 immunization. Neurology 2003, 61(1):46-54.

7. Kloppenborg RP, Richard E, Sprengers ME, Troost D, Eikelenboom P, Nederkoorn PJ: Steroid responsive encephalopathy in cerebral amyloid angiopathy: a case report and review of evidence for immunosuppressive treatment. J Neuroinflammation 2010, 7:18.

8. Schwab P, Lidov HG, Schwartz RB, Anderson RJ: Cerebral amyloid angiopathy associated with primary angiitis of the central nervous system: report of 2 cases and review of the literature. Arthritis Rheum 2003, 49(3):421-427.

9. Greenberg SM, Rapalino O, Frosch MP: Case records of the Massachusetts General Hospital. Case 22-2010. An 87-year-old woman with dementia and a seizure. N Engl J Med 2010, 363(4):373-381.

10. Greenberg SM, Vonsattel JP, Stakes JW, Gruber M, Finklestein SP: The clinical spectrum of cerebral amyloid angiopathy: presentations without lobar hemorrhage. Neurology 1993, 43(10):2073-2079.
11. Ortiz O, Reed L: Cerebral amyloid angiopathy presenting as a nonhemorrhagic, infiltrating mass. Neuroradiology 1996, 38(5):449-452.

12. Fountain NB, Eberhard DA: Primary angiitis of the central nervous system associated with cerebral amyloid angiopathy: report of two cases and review of the literature. Neurology 1996, 46(1):190-197.

13. Anders KH, Wang ZZ, Kornfeld M, Gray F, Soontornniyomkij V, Reed LA, Hart MN, Menchine M, Secor DL, Vinters HV: Giant cell arteritis in association with cerebral amyloid angiopathy: immunohistochemical and molecular studies. Hum Pathol 1997, 28(11):1237-1246.

14. Fountain NB, Lopes MB: Control of primary angiitis of the CNS associated with cerebral amyloid angiopathy by cyclophosphamide alone. Neurology 1999, 52(3):660-662.

15. Case records of the Massachusetts General Hospital. Weekly clinicopathological exercises. Case 10-2000. A 63-year-old man with changes in behavior and ataxia. N Engl J Med 2000, 342(13):957-965.

16. Oide T, Tokuda T, Takei Y, Takahashi H, Ito K, Ikeda S: Serial CT and MRI findings in a patient with isolated angiitis of the central nervous system associated with cerebral amyloid angiopathy. Amyloid 2002, 9(4):256-262.

17. Tamargo RJ, Connolly ES, McKhann GM, Khandji A, Chang Y, Libien J, Adams D: Clinicopathological review: primary angiitis of the central nervous system in association with cerebral amyloid angiopathy. Neurosurgery 2003, 53(1):136-143, discussion 143

18. Safriel Y, Sze G, Westmark K, Baehring J: MR spectroscopy in the diagnosis of cerebral amyloid angiopathy presenting as a brain tumor. AJNR Am J Neuroradiol 2004, 25(10):1705-1708.

19. Hashizume Y, Yoshida M, Suzuki E, Hirayama M: A 65-year-old man with headaches and left homonymous hemianopsia. Neuropathology 2004, 24(4):350-353.

20. Harkness KA, Coles A, Pohl U, Xuereb JH, Baron JC, Lennox GG: Rapidly reversible dementia in cerebral amyloid inflammatory vasculopathy. Eur J Neurol 2004, 11(1):59-62.

21. Jacobs DA, Liu GT, Nelson PT, Galetta SL: Primary central nervous system angiitis, amyloid angiopathy, and Alzheimer's pathology presenting with Balint's syndrome. Surv Ophthalmol 2004, 49(4):454-459.

22. Mikolaenko I, Conner MG, Jinnah HA: A 50-year-old man with acute-onset generalized seizure. Cerebral amyloid angiopathy and associated giant cell reaction. Arch Pathol Lab Med 2006, 130(1):e5-7.

23. Wong SH, Robbins PD, Knuckey NW, Kermode AG: Cerebral amyloid angiopathy presenting with vasculitic pathology. J Clin Neurosci 2006, 13(2):291-294.

24. Greenberg SM, Parisi JE, Keegan BM: A 63-year-old man with headaches and behavioral deterioration. Neurology 2007, 68(10):782-787.

25. Marotti JD, Savitz SI, Kim WK, Williams K, Caplan LR, Joseph JT: Cerebral amyloid angiitis processing to generalized angiitis and leucoencephalitis. Neuropathol Appl Neurobiol 2007, 33(4):475-479.

26. McHugh JC, Ryan AM, Lynch T, Dempsey E, Stack J, Farrell MA, Kelly PJ: Steroid-responsive recurrent encephalopathy in a patient with cerebral amyloid angiopathy. Cerebrovasc Dis 2007, 23(1):66-69.

27. Takeda A, Tatsumi S, Yamashita M, Yamamoto T: [Granulomatous angiitis of the CNS associated with cerebral amyloid angiopathy-an autopsied case with widespread involvement]. Brain Nerve 2007, 59(5):537-543.

28. Machida K, Tojo K, Naito KS, Gono T, Nakata Y, Ikeda S: Cortical petechial hemorrhage, subarachnoid hemorrhage and corticosteroid-responsive leukoencephalopathy in a patient with cerebral amyloid angiopathy. Amyloid 2008, 15(1):60-64.

29. Salvarani C, Brown RD, Calamia KT, Christianson TJ, Huston J, Meschia JF, Giannini C, Miller DV, Hunder GG: Primary central nervous system vasculitis: comparison of patients with and without cerebral amyloid angiopathy. Rheumatology (Oxford) 2008, 47(11):1671-1677.

30. Amick A, Joseph J, Silvestri N, Selim M: Amyloid-beta-related angiitis: a rare cause of recurrent transient neurological symptoms. Nat Clin Pract Neurol 2008, 4(5):279-283.

31. Alcalay RN, Smith EE: MRI showing white matter lesions and multiple lobar microbleeds in a patient with reversible encephalopathy. J Neuroimaging 2009, 19(1):89-91.

32. Daniels R, Geurts JJ, Bot JC, Schonewille WJ, van Oosten BW: Steroidresponsive edema in CAA-related inflammation. J Neurol 2009, 256(2):285-286.

33. Morishige M, Abe T, Kamida T, Hikawa T, Fujiki M, Kobayashi H, Okazaki T, Kimura N, Kumamoto T, Yamada A, et al: Cerebral vasculitis associated 
with amyloid angiopathy: case report. Neurol Med Chir (Tokyo) 2010, 50(4):336-338.

34. Savoiardo M, Erbetta A, Storchi G, Girotti F: Case 159: cerebral amyloid angiopathy-related inflammation. Radiology 2010, 256(1):323-327.

35. Cano LM, Martinez-Yelamos S, Majos C, Alberti MA, Boluda S, Velasco R, Rubio F: Reversible acute leukoencephalopathy as a form of presentation in cerebral amyloid angiopathy. J Neurol Sci 2010, 288(12):190-193.

36. DiFrancesco JC, Brioschi M, Brighina L, Ruffmann C, Saracchi E,

Costantino G, Galimberti G, Conti E, Curto NA, Marzorati L, et al: Anti-Abeta autoantibodies in the CSF of a patient with CAA-related inflammation: a case report. Neurology 2011, 76(9):842-844.

37. Chung KK, Anderson NE, Hutchinson D, Synek B, Barber PA: Cerebral amyloid angiopathy related inflammation: three case reports and a review. J Neurol Neurosurg Psychiatry 2011, 82(1):20-26.

doi:10.1186/1742-2094-8-116

Cite this article as: Sakaguchi et al:: Cerebral Amyloid Angiopathyrelated Inflammation Presenting with Steroid-responsive Higher Brain Dysfunction: Case Report and Review of the Literature. Journal of Neuroinflammation 2011 8:116.

\section{Submit your next manuscript to BioMed Central} and take full advantage of:

- Convenient online submission

- Thorough peer review

- No space constraints or color figure charges

- Immediate publication on acceptance

- Inclusion in PubMed, CAS, Scopus and Google Scholar

- Research which is freely available for redistribution

Submit your manuscript at www.biomedcentral.com/submit 